\title{
Research on Optimization of Aircraft Vertical Tail Stealth Design Based on Airborne PD Radar
}

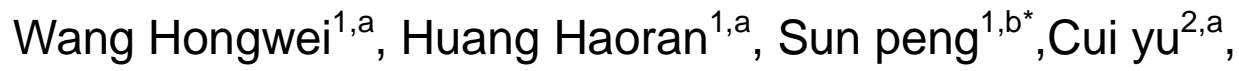 \\ Jiang Zhaochun ${ }^{3, a}$,Liang shicong ${ }^{4, a}$ \\ ${ }^{1}$ Department of Aircraft and Dynamics, The Aviation University of Air Force,Changchun \\ 130022,China \\ ${ }^{2}$ Equipment department of Shenyang Air Force, Shenyang, 110000,China \\ ${ }^{3 .}$ Representative Office in Qingdao Area of Air force, Qingdao 266000,China \\ ${ }^{4}$ Equipment department of No.94836 Troops , Nancang, 330201,China) \\ awhw1001@126.com, brocy527@163.com
}

Keywords: Stealth; optimization design; Kriging model; penetration fight

Abstract:To solve optimization of aircraft vertical tail stealth design under airborne PD radar, the Kriging model based on agent tail stealth characteristics is proposed; Genetic algorithm is applied to optimization calculation based on the tail stealth agent model as objective function and the tail capacity as constraints. The calculation results show that: It is feasible to optimize the stealth design of vertical tail, which can better improve the aircraft lateral stealth performance under the condition of the penetration fight.

\section{Introduction}

Low altitude and low altitude penetration technology in modern war is widely used and achieved great success. At low altitude and low altitude, as electromagnetic wave propagation conditions worsen, and ground clutter interference wave, resulting in the common pulse constitution of land-based and shipboard radar cannot work normally. In order to counter the low altitude target effectively, people develop new radar constitution, namely pulse Doppler (PD) radar. Put it on the plane that rise into the air, thus greatly reduces the low blind to overcome the influence of ground bending of radar wave ray normal propagation, which for aircraft in low altitude penetration is undoubtedly a great threat[1]. So it is important to improve the stealth performance of aircraft in PD radar.

Vertical tail, as the largest contributor of aircraft lateral RCS , undoubtedly becomes one of the main threats to combat aircraft penetration. This paper in view of combat environment of a certain type of aircraft penetration, establishes the vertical tail plane parametric model , and construct the vertical tail stealth characteristics of agent model by using Kriging model. The application of genetic algorithm for optimization calculation.

\section{Parametric modeling}

This paper focuses on the vertical tail plane parameters and spatial parameters influence on the performance of the vertical tail. Because the tail requirements are able to work in a positive and negative sideslip angle, airfoil using symmetrical airfoil. In this paper, based on a certain type of aircraft as the reference, a certain type airfoil of Russia is seleted. 


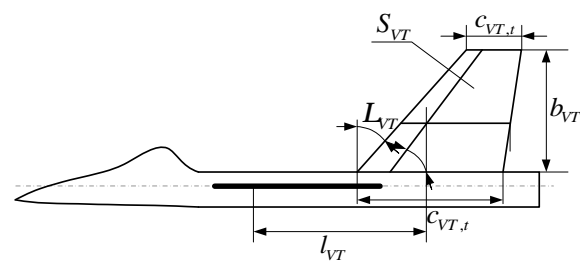

Figure1 The plane parameter of vertical tail

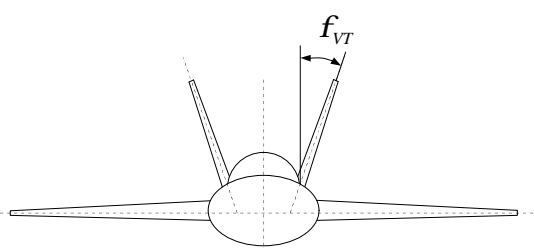

Figure2 The inclined angle of vertical tail

Through the analysis in Figure1, Figure2, we can find that a complete vertical tail can be determined by determining the leading-edge sweep angle $A_{V T}$, aspect ratio (single vertical tail) $A_{V T}$, relative area $R_{S, V T}$, ratio of root to shoot $\lambda_{V T}$, inclined angle $\phi_{V T}$ of vertical tail five parameters. Therefore, in this paper, $\Lambda_{V T}, A_{V T}, R_{S, V T}, \lambda_{V T}$ and $\phi_{V T}$ are defined as the vertical tail design variables, according to the statistics of the current active aircraft vertical tail parameters to determine the following ranges: $30^{\circ}<\Lambda_{V T}<50^{\circ} ; 1.0<\Lambda_{V T}<2.0 ; 20 \%<R_{S, V T}<30 \% ; 2.0<\lambda_{V T}<5.0 ; 15^{\circ}<\phi_{V T}<30^{\circ}$.

In this paper, the vertical tail test scheme is made by uniform design method ${ }^{[4]}$. In order to ensure the sample number can reflect the overall distribution of the whole space, choose the uniform design table $U_{16}\left(16^{5}\right)$. The aircraft model generated by a scheme is shown in Figure 3.

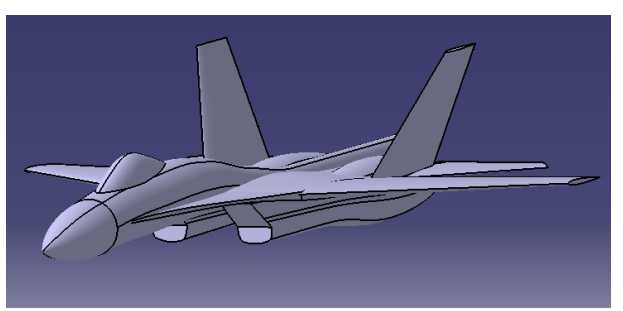

Figure3 A test model

\section{Optimization analysis}

For stealth optimization calculation of the vertical tail, in mathematics it can be expressed as:

Given parameters:

$$
f, \theta, \phi ;
$$

Design variables:

$$
\Lambda_{V T}, A_{V T}, R_{S, V T}, \lambda_{V T}, \phi_{V T}
$$

Objective function:

$$
\min f_{\sigma}\left(\Lambda_{V T}, A_{V T}, R_{S, V T}, \lambda_{V T}, \phi_{V T}\right)
$$

Restriction condition:

$$
\begin{aligned}
& f\left(\Lambda_{V T}, A_{V T}, R_{S, V T}, \lambda_{V T}, \phi_{V T}\right) \leq c \\
& 0.060<V_{V T}<0.10 .
\end{aligned}
$$

Where, $f, \theta, \phi$ are the radar frequency, the pitch angle and the azimuth range of the irradiation. $f\left(\Lambda_{V T}, A_{V T}, R_{S, V T}, \lambda_{V T}, \phi_{V T}\right) \leq c$ represents the range of the parameters $\Lambda_{V T}, A_{V T}, R_{S, V T}, \lambda_{V T}, \phi_{V T}$ in the second 
section. $V_{V T}$ is the tail boom capacity, is an important parameter affecting the static stability of the aircraft heading. Its size is relatively high and the product area and the tail wing span arm of the product, i.e.:

$$
V_{V T}=R_{S, V T} \frac{l_{V T}}{b_{W}}
$$

The actual situation of the current typical aircraft tail capacity. From the picture shows that its value is about 0.077 . So we take $0.060<V_{V T}<0.10$.

Because of the design variables of the optimization problem, the agent model of the target function is more complex, so the genetic algorithm is used to solve the problem. Genetic algorithm is based on the simulation of the process of biological evolution and evolution optimization algorithm, which has the characteristics of simple, universal, robust and strong, in dealing with nonlinear optimization problems show the good adaptability and flexibility.

The optimized results are shown in Table 1, and the distribution of the RCS with the range of the optimized shape is shown in Figure 4, for comparison with the initial scheme, and the initial scheme of the RCS is also given.

Table1 Optimization results

\begin{tabular}{|c|c|c|c|c|c|}
\hline Designvariable & $\Lambda_{V T}$ & $A_{V T}$ & $R_{S, V T}$ & $\lambda_{V T}$ & $\phi_{V T}$ \\
\hline $\begin{array}{c}\text { Before } \\
\text { optimization }\end{array}$ & $45^{\circ}$ & 1.5 & $24.2 \%$ & 3.91 & $0^{\circ}$ \\
\hline $\begin{array}{c}\text { After } \\
\text { optimization }\end{array}$ & $46.1^{\circ}$ & 1.36 & $26.67 \%$ & 4.18 & $13.96^{\circ}$ \\
\hline
\end{tabular}

Through the optimization design of aircraft vertical tail, the aircraft stealth performance has been significantly improved. After optimization, the RCS value $f_{\sigma}=9.876$ than that $f_{\sigma}=13.487$ before optimization reduced $26.6 \%$, and optimized the tail boom capacity $V_{V T}=0.0687$, basically meet the static stability of the aircraft heading, did not appear due to stealth performance improvement and lead to yaw statically unstable situation. This explains the through optimization of the Kriging model and genetic algorithm, the realization of the vertical tail shape stealth optimization design, lateral RCS of aircraft improved to stealth optimization purposes.

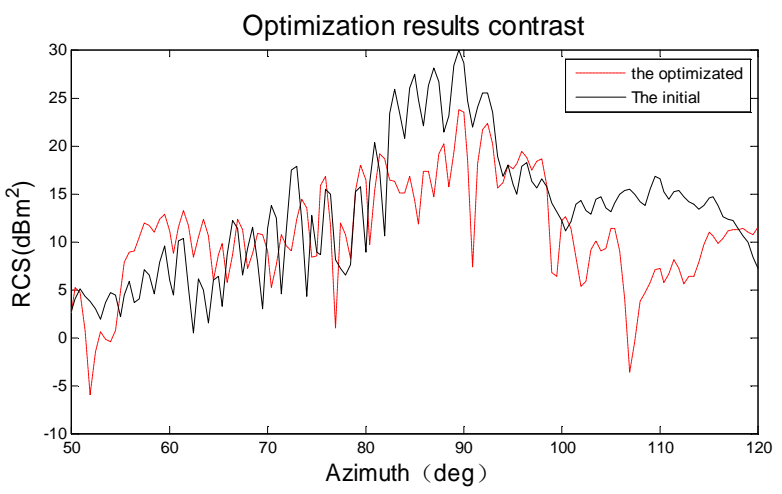

Figure4 Optimization results contrast 


\section{Summary}

In this paper a certain type of aircraft vertical tail shape, with vertical design samples based on uniform design to establish three-dimensional model. The plate edge element method is used to calculate RCS, construct the agent model of aircraft lateral RCS and tail design variables by using Kriging model, combine with the genetic algorithm, realizes the finned airplane stealth optimization design. The results show that based on the Kriging agent model has good accuracy and fast response speed; optimization of vertical tail design can improve the lateral aircraft stealth performance, at the same time to ensure the aircraft necessary directional static stability. This to improve our army aircraft penetration ability has important practical significance, also for China's existing aircraft stealth modification design provide a reference.

\section{Reference}

[1] Ye W,Zhu A H,Liu B,etal. Research on low-altitude penetration technologies of aircrafts. Electronics Optics and Control,2007,14 (4) 87-91.

[2] Ma K,Zhang Y F,Ma D L,etal. The aircraft on the radar stealth performance calculation and analysis. Beijing:National Defense Industry Press,2000:144-167.

[3] Mu X F, Yao W X,Yu X Q,etal..A survey of surrogate models used in MDO.Chinese Journal of Computational Mechanics,2005,22(5)608-612.

[4] Zeng Z J.Uniform Design and Application.Beijing:China Medicine Science and Technology Press,2005: 7-25. 